\title{
Efficiency of a pneumatic device in controlling cuff pressure of polyurethane-cuffed tracheal tubes: a randomized controlled study
}

Emmanuelle Jaillette ${ }^{1}$, Farid Zerimech ${ }^{2 \dagger}$, Julien De Jonckheere ${ }^{3 \dagger}$, Demosthenes Makris ${ }^{4}$, Malika Balduyck ${ }^{2,5}$, Alain Durocher ${ }^{1,6}$, Alain Duhamel ${ }^{7}$ and Saad Nseir ${ }^{1,6^{*}}$

\begin{abstract}
Background: The primary objective of this study was to determine the efficiency of a pneumatic device in controlling cuff pressure $\left(P_{\text {cuff }}\right)$ in patients intubated with polyurethane-cuffed tracheal tubes. Secondary objectives were to determine the impact of continuous control of $P_{\text {cuff, }}$ and cuff shape on microaspiration of gastric contents.

Methods: Prospective randomized controlled study. All patients requiring intubation and mechanical ventilation $\geq 48 \mathrm{~h}$ were eligible. The first 32 patients were intubated with tapered polyurethane-cuffed, and the 32 following patients were intubated with cylindrical polyurethane-cuffed tracheal tubes.

Patients randomly received $24 \mathrm{~h}$ of continuous control of $\mathrm{P}_{\text {cuff }}$ using a pneumatic device (Nosten ${ }^{\circledR}$ ), and $24 \mathrm{~h}$ of routine care of $\mathrm{P}_{\text {cuff }}$ using a manometer. Target $\mathrm{P}_{\text {cuff }}$ was $25 \mathrm{cmH}_{2} \mathrm{O}$. $\mathrm{P}_{\text {cuff }}$ was continuously recorded, and pepsin was quantitatively measured in all tracheal aspirates during these periods.

Results: The pneumatic device was efficient in controlling $\mathrm{P}_{\text {cuff }}$ (med $[\mathrm{IQ}] 26[24,28]$ vs $22[20,28] \mathrm{cmH}_{2} \mathrm{O}$, during continuous control of $\mathrm{P}_{\text {cuff }}$ and routine care, respectively; $\left.\mathrm{P}=0.017\right)$. In addition, percentage of patients with underinflation (31\% vs $68 \%$ ) or overinflation (53\% vs $100 \%$ ) of tracheal cuff, and percentage of time spent with underinflation $(0.9[0,17]$ vs $14 \%[4,30])$ or overinflation $(0[0,2]$ vs $32 \%[9,54])$ were significantly $(p<0.001)$ reduced during continuous control of $\mathrm{P}_{\text {cuff }}$ compared with routine care.

No significant difference was found in microaspiration of gastric content between continuous control of $P_{\text {cuff }}$ compared with routine care, or between patients intubated with tapered compared with cylindrical polyurethane-cuffed tracheal tubes.
\end{abstract}

Conclusion: The pneumatic device was efficient in controlling $\mathrm{P}_{\text {cuff }}$ in critically ill patients intubated with polyurethane-cuffed tracheal tubes.

Trial registration: The study was registered at clinicaltrial.gov (NCT01351259).

Keywords: Intubation, Polyurethane, Tracheal cuff, Microaspiration, Tracheal injury, Pneumonia

\footnotetext{
*Correspondence: s-nseir@chru-lille.fr

${ }^{\dagger}$ Equal contributors

${ }^{1}$ Critical Care Center, R. Salengro Hospital, University Hospital of Lille,

Rue E. Laine, 59037 Lille cedex, France

${ }^{6}$ Medical Assessment Laboratory, EA 2694, Nord de France University, 1 place

de Verdun, 59045 Lille, France

Full list of author information is available at the end of the article
} 


\section{Background}

Microaspiration of contaminated oropharyngeal secretions and gastric content frequently occurs in intubated critically ill patients, and plays a major role in the pathogenesis of ventilator-associated pneumonia (VAP) [1]. Aspiration of contaminated secretions is followed by tracheobronchial colonization that might progress into VAP depending on quantity, and virulence of microorganisms, and local and general defense mechanisms [2].

During the last decade, significant progress has been achieved in the field of prevention of microaspiration, and VAP $[3,4]$. Polyurethane-cuffed tracheal tubes were reported to significantly reduce leakage in in vitro studies, and microaspiration in intubated critically ill patients [5-7]. In addition, three clinical studies reported significant reduction in VAP, and nosocomial pneumonia rate in patients intubated with polyurethane-cuffed tracheal tubes compared with those intubated with standard polyvinyl chloride-cuffed tracheal tubes [8-10]. However, limitations of these studies, including the use of subglottic aspiration in the intervention group in one study, clinical definition of nosocomial pneumonia, and beforeafter design should be taken into account. Recent data coming from in vitro studies also suggested a beneficial effect of tapered-shaped tracheal cuff in reducing microaspiration [11-13].

Underinflation, and overinflation of tracheal cuff are major risk factors for microaspiration, VAP, and tracheal injury [14-16]. Despite routine control of cuff pressure $\left(\mathrm{P}_{\text {cuff }}\right)$ using a manometer, patients intubated with polyvinyl-chloride or polyurethane-cuffed tracheal tubes spend a large amount of time with underinflation and overinflation of tracheal cuff $[6,17]$. Continuous control of polyvinyl chloride-cuffed tracheal tubes using a pneumatic device was found to be associated with significantly reduced microaspiration of gastric content, and VAP incidence [18]. However, there are several differences between polyvinyl chloride and polyurethane, including thickness and physicochemical properties. Condensation formation in the pilot external balloon is very frequent in patients intubated with polyurethanecuffed tracheal tubes. Whether condensation formation or other physicochemical properties of polyurethane could influence the efficiency of a pneumatic device in continuously controlling $\mathrm{P}_{\text {cuff }}$ is unknown. To our knowledge, no study has evaluated the efficiency of a pneumatic or an electronic device in continuously controlling $\mathrm{P}_{\text {cuff }}$ in patients intubated with polyurethane-cuffed tracheal tubes. Therefore, we conducted this randomized controlled trial to determine the efficiency of a pneumatic device in controlling $\mathrm{P}_{\text {cuff }}$ in critically-ill patients intubated with polyurethane-cuffed tracheal tube compared with routine care using a manual manometer. The secondary objectives were to determine the impact of continuous control of
$\mathrm{P}_{\text {cuff, }}$ and cuff shape, i.e. tapered versus cylindrical, on microaspiration of gastric content.

\section{Methods}

The local institutional review board of the Lille University Hospital approved this study. The patients provided their written consent before randomization. In unconscious patients who were not able to give consent for inclusion in the study at randomization, relatives (next-of-kin) gave assent on every patient's behalf, and patients were later given the opportunity to withdraw from the study. The study was registered at clinicaltrial.gov (NCT01351259).

\section{Study design}

This prospective randomized controlled cross-over study was conducted in a single 10-bed medical ICU during a 17 -month period. Inclusion criteria were age $>18$ years, intubation in the ICU, and expected duration of invasive mechanical ventilation $\geq 48$ hours. Exclusion criteria were enrolment in another trial, contraindication for semirecumbent position or for enteral nutrition, and intubation before ICU admission.

Primary objective was to determine the efficiency of a pneumatic device in controlling $\mathrm{P}_{\text {cuff }}$ in patients intubated with polyurethane-cuffed tracheal tubes compared with routine care using a manual manometer. Secondary objectives were to determine the impact of continuous control of $\mathrm{P}_{\text {cuff, }}$ and cuff shape, i.e. tapered versus cylindrical, on microaspiration of gastric contents.

All patients were intubated with polyurethane-cuffed tracheal tubes. Tracheal tube size was 7.5 and 8 in women and men, respectively. The first 32 patients were intubated with a tapered-cuffed tracheal tube (SealGuard ${ }^{\oplus}$, Mallinckrodt, Athlone, Ireland), and the 32 following patients were intubated with a cylindrical-cuffed tracheal tube (Microcuff ${ }^{\oplus}$, Kimberly-Clark, Georgia, USA). Patients were randomly assigned to receive continuous control of $\mathrm{P}_{\text {cuff }}$ for 24 hours followed by routine care for 24 hours, or routine care for 24 hours followed by continuous control $\mathrm{P}_{\text {cuff }}$ for 24 hours. The target $\mathrm{P}_{\text {cuff was }} 25 \mathrm{~cm} \mathrm{H} \mathrm{H}_{2} \mathrm{O}$ during the two 24-hour periods. A computer-generated random assignment list in balanced blocks of four was used. Sealed opaque individual envelopes containing treatment assignments were numbered sequentially. All caregivers were blinded to the randomization schedule and the block size. Because of the nature of the intervention, physicians and nurses could not be blinded to the randomization arm. However, engineer who performed the analysis of $\mathrm{P}_{\text {cuff }}$ recording (JD) and physicians who performed pepsin measurement (FZ, and $\mathrm{MB}$ ) were blinded to study group assignment. 


\section{Tracheal cuff management}

Routine care of tracheal cuff was managed according to an internal procedure adapted from the Société de Réanimation de Langue Française recommendations [19]. A manual manometer (Ambu ${ }^{\circledR}$ Cuff Pressure Gauge, Ambu A/S, Ballerup, Denmark) was used to check and adjust $\mathrm{P}_{\text {cuff }}$ every 8 hours. Continuous control of $\mathrm{P}_{\text {cuff }}$ was performed using a pneumatic device (Nosten ${ }^{\oplus}$, Leved, St-Maur, France) [20].

\section{Outcome measurement}

In all patients, $\mathrm{P}_{\text {cuff }}$ and airway pressure were continuously recorded (Physiotrace ${ }^{\circledR}, \mathrm{CHRU}$, Lille, France) at a digitizing frequency of $100 \mathrm{~Hz}$ during the 48 hours following randomization, including 24 hours of continuous control of $\mathrm{P}_{\text {cuff, }}$ and 24 hours of routine care. Nurses were blinded to recording data. Pepsin was quantitatively measured in all tracheal aspirates during the same two 24-hour periods. In order to avoid overlap in pepsin results between the two 24-hour periods, tracheal suctioning was always performed at the end of the first periods of $\mathrm{P}_{\text {cuff }}$ control. Tracheal aspirates were stored at $-20^{\circ} \mathrm{C}$. Quantitative pepsin measurement was performed by an ELISA technique [18].

\section{Study population}

Measures aiming at preventing microaspiration were used in all patients including protocolized enteral nutrition, and sedation, minimal positive end expiratory pressure of $5 \mathrm{~cm} \mathrm{H}_{2} \mathrm{O}$, and semirecumbent position. Continuous subglottic suctioning was not utilized. Nurses performed tracheal suctioning every 3 hours or more if clinically indicated, using an open tracheal suction system.

\section{Definitions}

The primary end point was the percentage of patients with underinflation $\left(\mathrm{P}_{\text {cuff }}<20 \mathrm{~cm}\right) \mathrm{H}_{2} \mathrm{O}$ or overinflation ( $\mathrm{P}_{\text {cuff }}>30 \mathrm{~cm} \mathrm{H} \mathrm{H}_{2} \mathrm{O}$ ) of tracheal cuff. Secondary outcomes included duration of underinflation and overinflation of

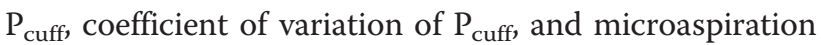
of gastric content. $\mathrm{P}_{\text {cuff }}$ continuous recording data were used to determine the time spent with normal $\mathrm{P}_{\text {cuff, }}$ underinflation, and overinflation of tracheal cuff. The coefficient of variation of $\mathrm{P}_{\text {cuff }}$ was calculated as standard deviation/mean $\mathrm{P}_{\text {cuff }} \times 100$. Microaspiration of gastric content was defined by the presence of pepsin at significant level $(>200 \mathrm{ng} / \mathrm{mL})$ in at least one tracheal aspirate.

\section{Statistical analyses}

Based on the incidence of underinflation or overinflation of tracheal cuff in patients intubated with polyurethane-cuffed tracheal tubes and receiving routine care of $\mathrm{P}_{\text {cuff }}$ in our ICU (70\%), we estimated an incidence of underinflation or overinflation in patients intubated with polyurethane-cuffed tracheal tubes and receiving continuous control of $\mathrm{P}_{\text {cuff }}$ of $30 \%$. Randomly assigning 32 patients to two 24-hour periods of Pcuff control would allow detection of this difference with $80 \%$ power and a two-tailed significance level of 0.05. To determine the impact of cuff shape on microaspiration of gastric content, two groups of 32 patients intubated with tapered or cylindrical-cuffed tracheal tubes were required.

All $P$ values were two-tailed. Categorical variables were described as frequencies (\%). Because they were not normally distributed, continuous variables were described as median (interquartile range). McNemar's test and Wilcoxon rank test were used to compare qualitative and quantitative variables between the two 24-hour periods, respectively. For comparisons between subgroups (tapered versus cylindrical-cuffed tracheal tubes), $x^{2}$ test or Fisher exact test were used to compare qualitative variables, and Mann-Whitney $U$ test was used to compare continuous variables.

To determine the impact of high airway pressures on the efficiency of the pneumatic device, we compared the time spent with underinflation and overinflation of $\mathrm{P}_{\text {cuff }}$ during continuous control of tracheal cuff between patients with high airway pressures $\left(\geq 75^{\text {th }}\right.$ quartile of airway pressures in the cohort), and those with lower airway pressures $\left(<75^{\text {th }}\right.$ quartile of airway pressures in the cohort).

\section{Results}

\section{Patient characteristics}

Sixty four patients were included in this study, including 32 intubated with tapered-cuffed tracheal tube, and 32 with cylindrical-cuffed tracheal tube. Study flow chart is presented in Figure 1. Median time from intubation to randomization was 3 days $(1,7)$. Median tracheal tube size was $8 \mathrm{~mm}$ (7.5-8). No significant difference was found in median time from intubation to randomization $(4[1,8]$ vs 2 days $[1,5]), \mathrm{p}=0.470$; or tracheal tube size (8 [7.5-8] vs $8 \mathrm{~mm}$ [7.5-8], p=0.456) between patients intubated with tapered-cuffed tracheal tubes and those intubated with cylindrical-cuffed tracheal tubes, respectively. No significant difference was found in patient characteristics between the two periods of continuous control of $\mathrm{P}_{\text {cuff }}$ and routine care, or between patients intubated with tapered-cuffed tracheal tubes, and those intubated with cylindrical-cuffed tracheal tubes (Tables 1, and 2).

\section{Continuous control versus routine care of tracheal cuff}

The pneumatic device was efficient in controlling $\mathrm{P}_{\text {cuff. }}$ Percentage of patients with underinflation or overinflation of tracheal cuff, coefficient of variation of $\mathrm{P}_{\text {cuff, }}$ and percentage of time spent with underinflation and overinflation of tracheal cuff were significantly lower during 


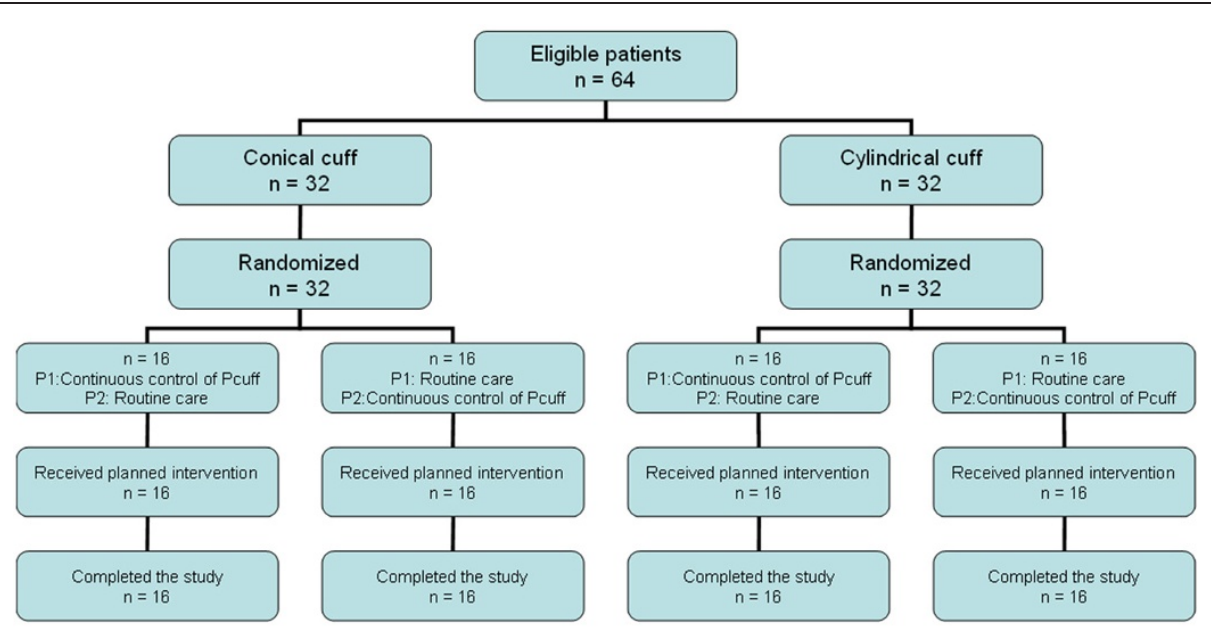

Figure 1 Study flowchart. Pcuff, cuff pressure; P1, period 1; P2, period 2.

continuous control of $\mathrm{P}_{\text {cuff }}$ compared with routine care. $\mathrm{P}_{\text {cuff, }}$, and percentage of time spent with $\mathrm{P}_{\text {cuff }} 20-30$ $\mathrm{cmH}_{2} \mathrm{O}$ were significantly higher during continuous control of $\mathrm{P}_{\text {cuff }}$ compared with routine control. No significant difference was found in rate of microaspiration of gastric content, mean pepsin level, or percentage of tracheal aspirates positive for pepsin between continuous control of $\mathrm{P}_{\text {cuff }}$ and routine care (Table 3). No significant interaction was found between continuous control of $\mathrm{P}_{\text {cuff }}$ and cuff shape $(\mathrm{p}=0.93)$.

Table 1 Patient characteristics at ICU admission

\begin{tabular}{|c|c|c|c|c|}
\hline Variables & $\begin{array}{c}\text { All patients } \\
n=64\end{array}$ & $\begin{array}{c}\text { Tapered cuff } \\
n=32\end{array}$ & $\begin{array}{c}\text { Cylindrical cuff } \\
n=32\end{array}$ & $\mathrm{P}^{*}$ \\
\hline Age & $66(55-75)$ & $66(58-77)$ & $66(54-72)$ & 0.330 \\
\hline Male gender & $43(67)$ & $20(62)$ & $23(71)$ & 0.594 \\
\hline SAPS ॥ & $45(32-62)$ & $45(29-62)$ & $42(37-62)$ & 0.289 \\
\hline LOD score & $6(3-9)$ & $6(2-10)$ & $6(4-9)$ & 0.818 \\
\hline \multicolumn{5}{|l|}{ Comorbidities } \\
\hline Diabetes mellitus & $16(25)$ & $10(31)$ & $6(18)$ & 0.386 \\
\hline COPD & $23(35)$ & $11(34)$ & $12(37)$ & $>0.999$ \\
\hline Cardiovascular disease & $12(18)$ & $7(21)$ & $5(15)$ & 0.749 \\
\hline Cirrhosis & $1(1)$ & $1(3)$ & 0 & $>0.999$ \\
\hline \multicolumn{5}{|l|}{ Causes for ICU admission } \\
\hline Acute exacerbation of COPD & $18(28)$ & $10(31)$ & $8(25)$ & 0.781 \\
\hline ARDS & $7(10)$ & $3(9)$ & $4(12)$ & $>0.999$ \\
\hline Septic shock & $15(23)$ & $10(31)$ & $5(15)$ & 0.238 \\
\hline Community-acquired pneumonia & $19(29)$ & $8(25)$ & $11(34)$ & 0.584 \\
\hline Hospital acquired pneumonia & $24(37)$ & $16(50)$ & $8(25)$ & 0.071 \\
\hline Congestive heart failure & $4(6)$ & $2(6)$ & $2(6)$ & $>0.999$ \\
\hline Neurologic failure & $7(10)$ & $2(6)$ & $5(15)$ & 0.426 \\
\hline Poisoning & $3(4)$ & $2(6)$ & $1(3)$ & $>0.999$ \\
\hline Soft tissue infetion & $2(3)$ & 0 & $2(6)$ & 0,492 \\
\hline
\end{tabular}

Data are frequencies (\%) or median (Interquartile range).

SAPS, simplified acute physiology score; LOD, logistic organ dysfunction; COPD, chronic obstructive pulmonary disease; ARDS, acute respiratory distress syndrome. Some patients had more than one cause for ICU admission.

*P values are for comparisons of tapered with cylindrical cuff. 
Table 2 Patient characteristics during the $\mathbf{4 8}$ hours following randomization

\begin{tabular}{|c|c|c|c|c|c|c|c|c|c|}
\hline \multirow[t]{2}{*}{ Variables } & \multirow[t]{2}{*}{$\begin{array}{l}\text { Continuous control of } P_{\text {cuff }} \\
\qquad n=64\end{array}$} & \multirow[t]{2}{*}{$\begin{array}{l}\text { Routine care } \\
n=64\end{array}$} & \multirow[t]{2}{*}{$p$} & \multicolumn{2}{|c|}{$\begin{array}{l}\text { Continuous control of } P_{\text {cuff }} \\
n n=64\end{array}$} & \multirow[t]{2}{*}{$p$} & \multicolumn{2}{|c|}{$\begin{array}{c}\text { Routine care } \\
n=64\end{array}$} & \multirow[t]{2}{*}{$p$} \\
\hline & & & & $\begin{array}{c}\text { Tapered } \\
\text { cuff } \\
\mathrm{n}=32\end{array}$ & $\begin{array}{c}\text { Cylindrical } \\
\text { cuff } \\
n=32\end{array}$ & & $\begin{array}{c}\text { Tapered } \\
\text { cuff } \\
n=32\end{array}$ & $\begin{array}{c}\text { Cylindrical } \\
\text { cuff } \\
n=32\end{array}$ & \\
\hline \multirow{2}{*}{$\begin{array}{l}\text { Quantity of enteral } \\
\text { nutrition, } \mathrm{ml} / \text { day }\end{array}$} & 1000 & 1000 & 0.433 & 1000 & 1000 & 0.960 & 1000 & 1000 & 0.241 \\
\hline & $(500-1000)$ & $(500-1500)$ & & $(500-1000)$ & $(500-1000)$ & & $(500-1000)$ & $(500-1500)$ & \\
\hline $\begin{array}{l}\text { Gastric residual } \\
\text { volume, } \mathrm{ml}\end{array}$ & $0(0-20)$ & $0(0-20)$ & 0.871 & $0(0-15)$ & $0(0-27)$ & 0.726 & $0(0-60)$ & $0(0-5)$ & 0.294 \\
\hline Sedation & $28(43)$ & $29(45)$ & $>0.999$ & $14(43)$ & $14(43)$ & $>0.999$ & $15(46)$ & $14(43)$ & $>0.999$ \\
\hline Ramsay score & $2(2-3)$ & $2(2-3)$ & 0.641 & $2(2-3)$ & $2(2-3)$ & 0.718 & $2(2-3)$ & $2(2-3)$ & 0.729 \\
\hline $\begin{array}{l}\text { Neuromuscular } \\
\text { blocking agent use }\end{array}$ & $6(9)$ & $7(10)$ & $>0.999$ & $3(9)$ & $3(9)$ & $>0.999$ & $3(9)$ & $4(12)$ & $>0.999$ \\
\hline Glasgow coma score & $14(10-15)$ & $15(10-15)$ & 0.310 & $13(10-15)$ & $14(8-15)$ & 0.738 & $14(10-15)$ & $15(8-15)$ & 0.908 \\
\hline PEEP & $6(5-8)$ & $6(5-9)$ & 0.943 & $6(5-8)$ & $7(5-9)$ & 0.234 & $6(5-9)$ & $7(5-9)$ & 0.166 \\
\hline Ventilatory mode & & & 0.329 & & & 0.885 & & & 0.433 \\
\hline $\mathrm{ACV}$ & $36(56)$ & $37(57)$ & & $18(56)$ & $18(56)$ & & $21(65)$ & $16(50)$ & \\
\hline PSV & $23(35)$ & $25(39)$ & & $11(34)$ & $12(37)$ & & $10(31)$ & $15(46)$ & \\
\hline BPPV & $5(7)$ & $2(3)$ & & $3(9)$ & $2(6)$ & & $1(3)$ & $1(3)$ & \\
\hline $\begin{array}{l}\text { Stress ulcer prophylaxis } \\
\text { or treatment }\end{array}$ & & & $>0.999$ & & & 0.688 & & & 0.109 \\
\hline Sucralfate & $32(50)$ & $32(50)$ & & $15(46)$ & $17(53)$ & & $13(40)$ & $19(59)$ & \\
\hline $\begin{array}{l}\text { Proton pump } \\
\text { inhibitors }\end{array}$ & $25(39)$ & $25(39)$ & & $13(40)$ & $12(37)$ & & $13(40)$ & $12(37)$ & \\
\hline Prokinetic drugs & $12(18)$ & $12(18)$ & $>0.999$ & $6(18)$ & $6(18)$ & $>0.999$ & $7(21)$ & $5(15)$ & 0.749 \\
\hline Vomiting & $3(4)$ & $1(1)$ & 0.625 & 0 & $3(9)$ & 0.237 & $1(3)$ & 0 & $>0.999$ \\
\hline Head-of-bed position & $40(35-40)$ & $40(35-40)$ & 0.846 & $40(35-45)$ & $40(35-40)$ & 0.602 & $40(35-40)$ & $40(35-45)$ & 0.244 \\
\hline
\end{tabular}

Data are frequencies (\%) or median (Interquartile range).

$\mathrm{P}_{\text {cuff, }}$ cuff pressure; PEEP, positive end-expiratory pressure; ACV, assist-control ventilation; PSV, pressure support ventilation; BPPV, bilevel positive pressure ventilation.

Tapered versus cylindrical cuff shape during continuous control of $\mathrm{P}_{\text {cuff }}$

During continuous control of $\mathrm{P}_{\text {cuff, }}$ percentage of patients with underinflation of tracheal cuff $>30$ minutes was significantly lower in patients intubated with tapered-cuffed tracheal tubes compared with those intubated with cylindrical-cuffed tracheal tubes. No significant difference was found in terms of $\mathrm{P}_{\text {cuff, }}$ percentage of patients with underinflation or overinflation, coefficient of variation of $\mathrm{P}_{\text {cuff, }}$ and percentage of time spent with underinflation or overinflation between the two groups during the same period (Table 4).

Tapered versus cylindrical cuff shape during routine care of $P_{\text {cuff }}$

During routine care, percentage of time spent with underinflation of tracheal cuff was significantly higher, and percentage of time spent with overinflation of tracheal cuff was significantly lower in patients intubated with tapered-cuffed tracheal tubes compared with those intubated with cylindrical-cuffed tracheal tubes. No significant difference was found in coefficient of variation of $\mathrm{P}_{\text {cuff }}$, or percentage of patients with underinflation or overinflation of tracheal cuff between the two groups during the same period (Table 4).

\section{Impact of cuff shape on microaspiration}

No significant difference was found in microaspiration of gastric content rate, mean pepsin level or percentage of tracheal aspirates positive for pepsin between patients intubated with tapered-cuffed tracheal tubes and those intubated with cylindrical-cuffed tracheal tubes during continuous control of $\mathrm{P}_{\text {cuff }}$ or routine care (Table 4).

\section{Other results}

During continuous control of $\mathrm{P}_{\text {cuff, important condensa- }}$ tion was observed in the pilot balloon in 7 (22\%) patients intubated with tapered-cuffed tracheal tube compared with $10(31 \%)$ patients intubated with cylindrical-cuffed tracheal tube $(\mathrm{p}=0.571)$.

No significant difference was found in percentage of time spent with underinflation (median [IQR] 0.07\% [0.0004-16] versus $0.01 \%$ [0.0003-0.6], $\mathrm{p}=0.359)$, or overinflation of tracheal cuff (6\% [0.27-37] versus $0.34 \%$ 
Table 3 Impact of the pneumatic device on tracheal cuff pressure and microaspiration of gastric content

\begin{tabular}{|c|c|c|c|c|}
\hline Variables & $\begin{array}{l}\text { Continuous control of } P_{\text {cuff }} \\
\qquad n=64\end{array}$ & $\begin{array}{c}\text { Routine care } \\
\mathrm{n}=64\end{array}$ & $p$ & OR $(95 \% \mathrm{Cl})$ \\
\hline Recording duration, $\mathrm{h}$ & $24(23-24)$ & $24(23-24)$ & 0.198 & \\
\hline Mean airway pressure, $\mathrm{cm} \mathrm{H}_{2} \mathrm{O}$ & $13(11-18)$ & $13(11-17)$ & 0.216 & \\
\hline Mean $\mathrm{P}_{\text {cuff, }} \mathrm{cm} \mathrm{H} \mathrm{H}_{2} \mathrm{O}$ & $26(24-28)$ & $22(20-28)$ & 0.017 & \\
\hline Coefficient of $\mathrm{P}_{\text {cuff }}$ variation, $\%$ & $5(3-12)$ & $19(15-30)$ & $<0.001$ & \\
\hline \multicolumn{5}{|l|}{$P_{\text {cuff }} 20-30 \mathrm{~cm} \mathrm{H}_{2} \mathrm{O}$} \\
\hline yes & $64(100)$ & $64(100)$ & NA & \\
\hline$\%$ of recording time & 95 (70-99) & $44(30-59)$ & $<0.001$ & \\
\hline \multicolumn{5}{|l|}{$P_{\text {cuff }}<20$ or $>30, \mathrm{~cm} \mathrm{H}_{2} \mathrm{O}$} \\
\hline Yes & $41(66)$ & $64(100)$ & $<0.001$ & \\
\hline Duration $>30 \mathrm{~min}$ & $33(51)$ & $64(100)$ & $<0.001$ & \\
\hline \multicolumn{5}{|l|}{$P_{\text {cuff }}<20, \mathrm{~cm} \mathrm{H}_{2} \mathrm{O}$} \\
\hline Yes & $19(29)$ & $60(93)$ & $<0.001$ & $1.46(1.23-1.73)$ \\
\hline Duration > $30 \mathrm{~min}$ & $16(25)$ & $57(89)$ & $<0.001$ & $2.63(1.11-6.21)$ \\
\hline$\%$ of recording time & $0.01(0-2)$ & $32(9-54)$ & $<0.001$ & \\
\hline \multicolumn{5}{|l|}{$P_{\text {cuff }}>30, \mathrm{~cm} \mathrm{H}_{2} \mathrm{O}$} \\
\hline Yes & $33(51)$ & $62(96)$ & 0.001 & $1.57(1.10-2.24)$ \\
\hline Duration $>30$ min & $25(39)$ & $54(84)$ & $<0.001$ & $1.50(1.11-2.20$ \\
\hline$\%$ of recording time & $0.9(0-17)$ & $14(4-30)$ & $<0.001$ & \\
\hline Microaspiration of gastric content & $32(50)$ & $38(59)$ & 0.238 & \\
\hline Pepsin, ng/mL & $185(113-296)$ & $203(120-338)$ & 0.171 & \\
\hline$\%$ of tracheal aspirates positive for pepsin & $29(0-74)$ & $45(0-100)$ & 0.162 & \\
\hline
\end{tabular}

Data are frequencies (\%) or median (Interquartile range). $P_{\text {cuff, }}$ cuff pressure.

Yes indicates that a patient had the variable at least once.

[0.02-11]) between patients with high airway pressures $(\mathrm{n}=16)$ and those with lower airway pressures $(\mathrm{n}=48)$, respectively.

\section{Discussion}

The percentage of patients with underinflation or overinflation of tracheal cuff, percentage of time spent with underinflation or overinflation of tracheal cuff, and coefficient of variation of $\mathrm{P}_{\text {cuff }}$ were significantly lower during continuous control of $\mathrm{P}_{\text {cuff }}$ compared with routine care. Further, percentage of time spent with $\mathrm{P}_{\text {cuff }} 20-30$ $\mathrm{cmH}_{2} \mathrm{O}$, and mean $\mathrm{P}_{\text {cuff }}$ were significantly higher during continuous control of $\mathrm{P}_{\text {cuff }}$ compared with routine care. However, no significant difference was found in the incidence of microaspiration of gastric content between continuous control of $\mathrm{P}_{\text {cuff }}$ compared with routine care or between patients intubated with tapered-cuffed tracheal tubes and those intubated with cylindrical-cuffed tracheal tubes.

To our knowledge, our study is the first to demonstrate the efficiency of the pneumatic device in controlling $\mathrm{P}_{\text {cuff }}$ in critically-ill patients intubated with polyurethane-cuffed tracheal tubes. Previous studies found similar results in animals and humans intubated with polyvinyl chloride- cuffed tracheal tubes [20,21]. The percentage of patients with underinflation of tracheal cuff $>30$ minutes during continuous control of $\mathrm{P}_{\text {cuff }}$ was relatively high (25\%). This could be explained by the physicochemical characteristics of polyurethane, namely its hydrophilic aspect, resulting in condensation formation especially during continuous

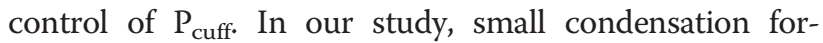
mation was rarely observed in the pilot balloon during routine care of $\mathrm{P}_{\text {cuff. However, important condensation }}$ formation filling the whole pilot balloon was frequently observed during continuous control of $\mathrm{P}_{\text {cuff. }}$ The fact that evaporation is probably reduced in closed circuit during continuous control of $\mathrm{P}_{\text {cuff }}$ might explain this difference in condensation formation between routine care and continuous control of $\mathrm{P}_{\text {cuff. }}$ Further studies directly comparing efficiency of the pneumatic device in polyurethane and polyvinyl chloride-cuffed tracheal tubes are needed to confirm our results. Although previous studies have demonstrated the efficiency of the pneumatic device in controlling $\mathrm{P}_{\text {cuff }}$ in patients intubated with PVC-cuffed tracheal tubes, the current study allow the generalization of this observation to patients intubated with polyurethane-cuffed tracheal tubes. Given the growing evidence supporting the use of polyurethane-cuffed tracheal tubes 
Table 4 Impact of cuff shape on cuff pressure and microaspiration during continuous control of cuff pressure and routine care

\begin{tabular}{|c|c|c|c|c|c|c|}
\hline \multirow[t]{2}{*}{ Variables } & \multicolumn{2}{|c|}{$\begin{array}{l}\text { Continuous control of } P_{\text {cuff }} \\
\qquad n=64\end{array}$} & \multirow[t]{2}{*}{$p$} & \multicolumn{2}{|c|}{$\begin{array}{c}\text { Routine care } \\
\mathrm{n}=64\end{array}$} & \multirow[t]{2}{*}{$p$} \\
\hline & $\begin{array}{c}\text { Tapered cuff } \\
\mathrm{n}=32\end{array}$ & $\begin{array}{c}\text { Cylindrical cuff } \\
n=32\end{array}$ & & $\begin{array}{c}\text { Tapered cuff } \\
\mathrm{n}=32\end{array}$ & $\begin{array}{c}\text { Cylindrical cuff } \\
n=32\end{array}$ & \\
\hline Recording duration, $\mathrm{h}$ & $24(23-24)$ & $24(23-25)$ & 0.662 & $24(23-25)$ & $24(23-24)$ & 0.229 \\
\hline Mean airway pressure, $\mathrm{cm} \mathrm{H}_{2} \mathrm{O}$ & $13(10-18)$ & $13(11-16)$ & 0.749 & $12(10-20)$ & $15(11-15)$ & 0.214 \\
\hline Mean $\mathrm{P}_{\text {cuff, }} \mathrm{cm} \mathrm{H} \mathrm{H}_{2} \mathrm{O}$ & $26(25-28)$ & $25(23-28)$ & 0.844 & $21(19-26)$ & $24(21-30)$ & 0.143 \\
\hline Coefficient of $P_{\text {cuff }}$ variation, $\%$ & $5(3-9)$ & $6(3-15)$ & 0.382 & $18(15-30)$ & $20(14-39)$ & 0.789 \\
\hline \multicolumn{7}{|l|}{$P_{\text {cuff }} 20-30 \mathrm{~cm} \mathrm{H}_{2} \mathrm{O}$} \\
\hline yes & $32(100)$ & $32(100)$ & NA & $32(100)$ & $32(100)$ & NA \\
\hline$\%$ of recording time & $97(82-99)$ & $88(56-99)$ & 0.147 & $42(30-62)$ & $44(29-57)$ & 0.811 \\
\hline \multicolumn{7}{|l|}{$P_{\text {cuff }}<20$ or $>30 \mathrm{~cm} \mathrm{H}_{2} \mathrm{O}$} \\
\hline Yes & $19(59)$ & $22(68)$ & 0.856 & $32(100)$ & $32(100)$ & $>0.999$ \\
\hline Duration $>30 \mathrm{~min}$ & $14(43)$ & $19(59)$ & 0.455 & $30(93)$ & $32(100)$ & $>0.999$ \\
\hline \multicolumn{7}{|l|}{$P_{\text {cuff }}<20 \mathrm{~cm} \mathrm{H}_{2} \mathrm{O}$} \\
\hline Yes & $6(18)$ & $13(40)$ & 0.116 & $29(90)$ & $31(96)$ & $>0.999$ \\
\hline Duration > 30 min & $3(9)$ & $13(40)$ & $0.011^{*}$ & $29(90)$ & $28(87)$ & 0.371 \\
\hline$\%$ of recording time & $0(0-0)$ & $0(0-12)$ & 0.549 & $41(17-62)$ & $21(8-47)$ & 0.049 \\
\hline \multicolumn{7}{|l|}{$P_{\text {cuff }}>30 \mathrm{~cm} \mathrm{H} \mathrm{H}_{2} \mathrm{O}$} \\
\hline Yes & $15(46)$ & $18(56)$ & 0.903 & $31(96)$ & $31(96)$ & $>0.999$ \\
\hline Duration $>30$ min & $12(37)$ & $13(40)$ & $>0.999$ & $24(75)$ & $30(93)$ & 0.235 \\
\hline$\%$ of recording time & $0.6(0-24)$ & $1.4(0-48)$ & 0.652 & $11(2-17)$ & $20(6-18)$ & 0.031 \\
\hline Microaspiration of gastric content & $13(40)$ & $19(59)$ & 0.211 & $18(56)$ & $20(62)$ & 0.799 \\
\hline Pepsin, ng/mL & $154(105-230)$ & $214(126-466)$ & 0.066 & $175(121-252)$ & $238(101-664)$ & 0.238 \\
\hline$\%$ of tracheal aspirates positive for pepsin & $0(0-57)$ & $43(0-100)$ & 0.073 & $40(0-86)$ & $50(0-100)$ & 0.310 \\
\hline
\end{tabular}

Data are frequencies (\%) or median (Interquartile range).

$\mathrm{P}_{\text {cuff, }}$ cuff pressure. Yes indicates that a patient had the variable at least once. ${ }^{*}$ OR $(95 \% \mathrm{Cl}) 3.20(1.12-9.12)$.

to prevent microaspiration and VAP, our results are necessary before performing further studies aiming to evaluate the combined beneficial effects of using polyurethanecuffed tracheal tubes and continuous control of $\mathrm{P}_{\text {cuff }}$ in preventing VAP.

In spite of efficient control of $\mathrm{P}_{\text {cuff, }}$ no significant difference was found in microaspiration of gastric content between continuous control of $\mathrm{P}_{\text {cuff }}$ and routine care. One potential explanation for this result is the optimal routine care provided during the study, and the use of polyurethane-cuffed tracheal tubes. These tubes have been demonstrated to significantly reduce microaspiration in critically ill patients [5,6]. Further, our study is probably underpowered to detect such an effect, since microaspiration of gastric content was a secondary outcome and the number of included patients was calculated based on the primary outcome. Further studies are needed to determine the impact of continuous control of $\mathrm{P}_{\text {cuff }}$ on the incidence of microaspiration, and VAP. However, whilst our study was underpowered to detect a difference in microaspiration, it was correctly powered to draw a valuable conclusion on primary outcome, i.e. the efficiency of the pneumatic device in controlling $\mathrm{P}_{\text {cuff. }}$ In fact, the number of patients required to detect the estimated difference in percentage of patients with underinflation or overinflation of $\mathrm{P}_{\text {cuff, }}$, with an alpha risk of $5 \%$ and a power of $80 \%$, was 32 per group. As 32 patients were included in each study group, our study is sufficiently powered to conclude that the pneumatic device is efficient in controlling $\mathrm{P}_{\text {cuff. }}$ This result would allow future studies to evaluate the impact of the pneumatic device on the prevention of complications related to underinflation and overinflation of $\mathrm{P}_{\text {cuff. Although our }}$ previous study has already suggested beneficial effects of continuous control of $\mathrm{P}_{\text {cuff }}$ in reducing microaspiration and VAP [18], it was conducted in patients intubated with PVC-cuffed tracheal tubes. Therefore, these results could not be generalized to patients intubated with polyurethane-cuffed tracheal tubes. Further studies with larger sample size are required in these patients to 
determine the impact of continuous control of $\mathrm{P}_{\text {cuff }}$ on prevention of cuff-related complications such as microaspiration, and VAP.

Some significant differences, including percentage of time with underinflation and overinflation were found between patients intubated with tapered-cuffed tracheal tubes compared with those intubated with cylindricalcuffed tracheal tubes. These differences could be explained by different cuff shape between the two groups. No significant effect was found of tracheal cuff shape on microaspiration of gastric content. However, during continuous control of $\mathrm{P}_{\text {cuff }}$ a trend towards lower pepsin level and percentage of tracheal aspirates positive for pepsin was found in patients intubated with tapered-cuffed tracheal tubes compared with those intubated with cylindricalcuffed tracheal tubes. Further, in spite of significantly higher percentage of time spent with underinflation of tracheal cuff in patients intubated with tapered-cuffed tracheal tubes compared with those intubated with cylindrical-cuffed tracheal tubes during routine care, microaspiration of gastric content rate was similar in the two groups. This result also suggests better sealing with tapered compared with cylindrical tracheal cuffs. However, our study was probably underpowered to detect a significant difference in microaspiration rate between the two groups.

Two recent in vitro studies found tapered-shaped tube cuff to considerably improve sealing characteristics of polyvinyl chloride tube cuffs $[11,13]$. However, no significant effect of the tapered-shaped cuff was found in polyurethane tube cuffs. In contrast, another in vitro study reported that tapered polyurethane cuff was more efficient than cylindrical polyurethane cuff in larger tracheal diameter in preventing fluid leakage [12]. Different study design might explain these conflicting results. In a prospective observational before-after study, our group found no significant difference in microaspiration of gastric content between patients intubated with tapered polyurethane-cuffed tracheal tubes compared with those intubated with cylindrical polyurethane-cuffed tracheal tubes [6]. Further clinical studies are required to determine the impact of tracheal cuff shape on the incidence of microaspiration, and VAP.

No significant difference was found in airway pressures between patients intubated with tapered compared with those intubated with cylindrical tracheal cuffs. Previous studies demonstrated that $\mathrm{P}_{\text {cuff }}$ was tightly correlated to airway pressure [22,23]. The relatively low airway pressures found in study patients were similar as those previously reported in other ICU patients $[6,24,25]$. This might strengthen our findings, since these results could be generalized to patients with similar airway pressure. Percentage of time spent with underinflation, and overinflation of $\mathrm{P}_{\text {cuff }}$ during continuous control of $\mathrm{P}_{\text {cuff }}$ was not significantly different between patients with high airway pressures and those with lower airway pressures, suggesting that the pneumatic device is efficient in patients with high airway pressures, such as those with severe asthma or ARDS. However, no definite conclusion could be drawn in this subgroup because of the small number of patients $(\mathrm{n}=16)$.

Our study has some limitations. First, this study was performed in a single ICU. Therefore, our results could not be generalized to other ICU patients. Second, our study did not evaluate the impact of continuous control of $\mathrm{P}_{\text {cuff }}$ on outcomes such as VAP or tracheal injury. However, in order to adjust for patient-related confounders, such as tracheal size and aspect, airway pressures, and tracheal tube size, continuous control and routine care of $\mathrm{P}_{\text {cuff }}$ were performed in the same patient. Therefore, it was not possible to evaluate these outcomes. Further, it was mandatory to evaluate the efficiency of the device in controlling $\mathrm{P}_{\text {cuff }}$ before performing studies on its impact on prevention of complications. Finally, randomization for tracheal cuff shape was performed per period and not per patient. However, intubation is often an urgent procedure. Therefore, it is very difficult to perform randomization per patient in such a study. In addition, the impact of cuff shape on microaspiration was a secondary outcome.

\section{Conclusion}

We conclude that the pneumatic device is efficient in controlling $\mathrm{P}_{\text {cuff }}$ in critically ill patients intubated with polyurethane-cuffed tracheal tube. Further studies are needed to determine the impact of continuous control of $\mathrm{P}_{\text {cuff }}$ and tracheal cuff shape on microaspiration, VAP, and tracheal injury.

\section{Key messages}

- The pneumatic device is efficient in controlling $\mathrm{P}_{\text {cuff }}$ in critically ill patients intubated with polyurethane-cuffed tracheal tube.

- Conical cuff shape could be beneficial in preventing microaspiration in critically ill patients.

- Further studies are needed to determine the impact of continuous control of $P_{\text {cuff }}$ and tracheal cuff shape on microaspiration, and VAP in patients intubated with polyurethane-cuffed tracheal tubes.

\section{Abbreviations}

ICU: Intensive care unit; $P_{\text {cuff: }}$ Cuff pressure; VAP: Ventilator-associated pneumonia.

\section{Competing interests}

SN: Covidien (advisory board), other authors: none.

\section{Authors' contribution}

EJ, ADur, and SN designed this study. EJ, FZ, JD, MB, and SN collected the data. ADuh performed statistical analyses. EJ, and SN wrote the manuscript, and all authors participated in its critical revision. EJ, and SN had full access to all data in the study and had final responsibility for the decision to submit for publication. All authors read and approved the final manuscript. 


\section{Acknowledgements}

The authors would like to thank Mrs. Catherine Lelorne, Mrs. Véronique Lemaire and Mrs. Nadine Parsy for their skilful assistance in pepsin measurement.

\section{Financial support}

Covidien and Kimberly-Clark donated tracheal tubes used in this study. None of these companies had any role in the design, study conduct, data analyses or reporting.

\section{Author details}

${ }^{1}$ Critical Care Center, R. Salengro Hospital, University Hospital of Lille, Rue E. Laine, 59037 Lille cedex, France. ${ }^{2}$ Biochemistry and Molecular Biology Laboratory, Biochemistry Division, Pathology and Biology Center, University Hospital of Lille, 59037 Lille cedex, France. ${ }^{3}$ Clinical Investigation Center Innovative Technologies, INSERM CIC-IT 807, University Hospital of Lille, 152 rue du Dr Alexandre Yersin, 59120 Loos, France. ${ }^{4}$ Intensive Care Unit, University Hospital of Larisa, University of Thessaly, Biopolis Street, 41110 Larisa, Greece. ${ }^{5}$ Biochemistry and Molecular Biology Laboratory, Faculty of Pharmacy, Lille II University, 1 place de Verdun, 59045 Lille, France. ${ }^{6}$ Medical Assessment Laboratory, EA 2694, Nord de France University, 1 place de Verdun, 59045 Lille, France. ${ }^{7}$ Epidemiology, Public Health and Quality of Care, Nord-de-France University, Lille, France.

Received: 31 March 2013 Accepted: 18 December 2013

Published: 26 December 2013

\section{References}

1. Nseir S, Zerimech F, Jaillette E, Artru F, Balduyck M: Microaspiration in intubated critically ill patients: diagnosis and prevention. Infect Disor Drug Target 2011, 11:413-423.

2. Craven DE, Chroneou A, Zias N, Hjalmarson Kl: Ventilator-associated tracheobronchitis: the impact of targeted antibiotic therapy on patient outcomes. Chest 2009, 135:521-528.

3. Bouadma L, Wolff M, Lucet J-C: Ventilator-associated pneumonia and its prevention. Curr Opin Infect Dis 2012, 25:395-404.

4. Coppadoro A, Bittner E, Berra L: Novel preventive strategies for ventilator-associated pneumonia. Crit Care 2012, 16:210.

5. Lucangelo U, Zin WA, Antonaglia V, Petrucci L, Viviani M, Buscema G, Borelli M, Berlot G: Effect of positive expiratory pressure and type of tracheal cuff on the incidence of aspiration in mechanically ventilated patients in an intensive care unit. Crit Care Med 2008, 36:409-413.

6. Nseir S, Zerimech F, De Jonckheere J, Alves I, Balduyck M, Durocher A Impact of polyurethane on variations in tracheal cuff pressure in critically ill patients: a prospective observational study. Intensive Care Med 2010, 36:1156-1163.

7. Dullenkopf A, Gerber A, Weiss M: Fluid leakage past tracheal tube cuffs: evaluation of the new Microcuff endotracheal tube. Intensive Care Med 2003, 29:1849-1853.

8. Lorente $L$, Lecuona $M$, Jiménez $A$, Mora ML, Sierra A: Influence of an endotracheal tube with polyurethane cuff and subglottic secretion drainage on pneumonia. Am J Respir Crit Care Med 2007, 176:1079-1083.

9. Poelaert J, Depuydt P, De Wolf A, Van de Velde S, Herck I, Blot S: Polyurethane cuffed endotracheal tubes to prevent early postoperative pneumonia after cardiac surgery: a pilot study. J Thor Cardiovascular Surg 2008, 135:771-776

10. Miller MA, Arndt JL, Konkle MA, Chenoweth CE, Iwashyna TJ, Flaherty KR, Hyzy RC: A polyurethane cuffed endotracheal tube is associated with decreased rates of ventilator-associated pneumonia. J Crit Care 2011, 26:280-286

11. Madjdpour C, Mauch J, Dave MH, Spielmann N, Weiss M: Comparison of air-sealing characteristics of tapered- vs. cylindrical-shaped high-volume, low-pressure tube cuffs. Acta Anaesthesio/ Scand 2012, 56:230-235.

12. Dave MH, Frotzler A, Spielmann N, Madjdpour C, Weiss M: Effect of tracheal tube cuff shape on fluid leakage across the cuff: an in vitro study. Brit J Anaesthesia 2010, 105:538-543.

13. Zanella A, Scaravilli V, Isgrò S, Milan M, Cressoni M, Patroniti N, Fumagalli $R$, Pesenti A: Fluid leakage across tracheal tube cuff, effect of different cuff material, shape, and positive expiratory pressure: a bench-top study. Intensive Care Med 2011, 37:343-347.
14. Young PJ, Rollinson M, Downward G, Henderson S: Leakage of fluid past the tracheal tube cuff in a benchtop model. Brit J Anaesthesia 1997. 78:557-562.

15. Rello J, Soñora R, Jubert P, Artigas A, Rué M, Vallés J: Pneumonia in intubated patients: role of respiratory airway care. Am J Respir Crit Care Med 1996, 154:111-115.

16. Seegobin RD, van Hasselt GL: Endotracheal cuff pressure and tracheal mucosal blood flow: endoscopic study of effects of four large volume cuffs. Brit Med J 1984, 288:965-968.

17. Nseir S, Brisson H, Marquette C-H, Chaud P, Di Pompeo C, Diarra M, Durocher A: Variations in endotracheal cuff pressure in intubated critically ill patients: prevalence and risk factors. Eur J Anaesthsio/ 2009, 26:229-234.

18. Nseir S, Zerimech F, Fournier C, Lubret R, Ramon P, Durocher A, Balduyck M: Continuous control of tracheal cuff pressure and microaspiration of gastric contents in critically ill patients. Am J Respir Crit Care Med 2011, 184:1041-1047.

19. Chastre J, Bedock B, Clair B, Gehanno P, Lacaze T, Lesieur O, Picart-Jacq JY, Plaisance $P$, Ravussin $P$, Samain E, et al: Quel abord trachéal pour la ventilation mécanique des malades de réanimation? (à l'exclusion du nouveau né). Reanimation 1998, 7:438-442.

20. Duguet A, D'Amico L, Biondi G, Prodanovic H, Gonzalez-Bermejo J, Similowski T: Control of tracheal cuff pressure: a pilot study using a pneumatic device. Intensive Care Med 2007, 33:128-132.

21. Nseir S, Duguet A, Copin M-C, De Jonckheere J, Zhang M, Similowski T, Marquette C-H: Continuous control of endotracheal cuff pressure and tracheal wall damage: a randomized controlled animal study. Crit Care 2007, 11:R109.

22. Guyton DC, Barlow MR, Besselievre TR: Influence of airway pressure on minimum occlusive endotracheal tube cuff pressure. Crit Care Med 1997 25:91-94.

23. Blanch PB: Laboratory evaluation of 4 brands of endotracheal tube cuff inflator. Respir Care 2004, 49:166-173.

24. Valta P, Corbeil C, Chassé M, Braidy J, Milic-Emili J: Mean airway pressure as an index of mean alveolar pressure. Am J Respir Crit Care Med 1996, 153:1825-1830.

25. Sole ML, Su X, Talbert S, Penoyer DA, Kalita S, Jimenez E, Ludy JE, Bennett $M$ : Evaluation of an intervention to maintain endotracheal tube cuff pressure within therapeutic range. Am J Crit Care 2011, 20:109-117.

doi:10.1186/1471-2253-13-50

Cite this article as: Jaillette et al:: Efficiency of a pneumatic device in controlling cuff pressure of polyurethane-cuffed tracheal tubes: a randomized controlled study. BMC Anesthesiology 2013 13:50.

\section{Submit your next manuscript to BioMed Central and take full advantage of:}

- Convenient online submission

- Thorough peer review

- No space constraints or color figure charges

- Immediate publication on acceptance

- Inclusion in PubMed, CAS, Scopus and Google Scholar

- Research which is freely available for redistribution 